\title{
Colonoscopia: Morbidade Negligenciada
}

\author{
Colonoscopy: Neglected Morbidity
}

\author{
FERNANDA BELLOTTIFORMIGA ${ }^{1}$; KARINA GOMES DAROCHA ${ }^{1}$; KARINADAGRE MAGRI ${ }^{1}$; MATHEUS PORTO \\ CARVALHO $^{1}$; ALESSANDRA VICENTINI CREDIDIO $^{\prime}$; SYLVIAHELOISAARANTES CRUZ $^{2}$; PAULODEAZEREDO \\ PASSOS CANDELÁRIA ${ }^{3}$; CHIABIN FANG ${ }^{4}$; PERETZCAPELHUCHNIK $^{5}$; WILMAR ARTUR KLUG ${ }^{5}$
}
${ }^{1}$ Residente da Disciplina de Coloproctologia da Santa Casa de São Paulo; ${ }^{2}$ Doutoranda da Faculdade de Ciências Médicas da Santa Casa de São Paulo; ${ }^{3}$ Mestre em Cirurgia; ${ }^{4}$ Professor Associado da Faculdade de Ciências Médicas da Santa Casa de São Paulo - Doutor em Cirurgia; ${ }^{5}$ Professor Titular da Faculdade de Ciências Médicas da Santa Casa de São Paulo.

\begin{abstract}
FORMIGA FB; ROCHA KG; MAGRI KD; CARVALHO MP; CREDIDIO AV; CRUZ SHA; CANDELÁRIA PAP; FANG CB; CAPELHUCHNIK P; KLUG WA. Colonoscopia: Morbidade Negligenciada. Rev bras Coloproct, 2009;29(3): 345-350.

RESUMO: Objetivo: Avaliar fatores de risco que determinam morbidade ao exame de colonoscopia. Métodos: No período de março a junho de 2009 foram analisados prospectivamente 170 pacientes submetidos a exame colonoscópico. Fatores de risco como idade, sexo, indicação, exame ambulatorial/internado, efeitos adversos e qualidade do preparo intestinal, procedimento endoscópico, diagnóstico e intercorrência peri-procedimento foram relacionados. Resultados: A média de idade da amostra foi 60,16 \pm 14,69 anos, com predominância do sexo feminino. A indicação mais prevalente do exame foi seguimento pós-operatório. Três exames foram inconclusivos por mau preparo. Do restante, 36,53\% foram normais e a maioria dos alterados apresentou pólipos, adenomatosos predominantemente. Quanto as comorbidades, 48,82\% dos pacientes possuíam alguma comorbidade, sendo Hipertensão Arterial Sistêmica a mais prevalente. Apenas $22,94 \%$ dos pacientes apresentaram algum efeito adverso ao preparo. O preparo foi limpo em $\mathbf{6 5 , 8 8 \%}$ dos exames, mostrando significância quando comparado a morbidade. Outro fator de significância estatística foi a realização de procedimentos $(44,7 \%$ dos exames), sendo a maioria polipectomias. A morbidade chegou a 16,47\%, sendo a desidratação a mais prevalente. Não houve mortalidade. Conclusão: A qualidade do preparo intestinal e a realização de procedimento endoscópico são fatores diretamente relacionados a morbidade do exame de colonoscopia.
\end{abstract}

Descritores: Colonoscopia, Efeitos adversos, Morbidade, Satisfação do paciente.

\section{INTRODUÇÃO}

A colonoscopia permite, desde a década de 60 , a visualização completa da mucosa do cólon e íleo terminal sendo considerado um método completo na investigação das doenças colorretais ${ }^{1}$. Atualmente através do desenvolvimento tecnológico com melhora na qualidade das imagens, nos procedimentos diagnósticos e terapêuticos como biópsias, polipectomias, mucosectomias, tatuagem de lesões suspeitas para posterior ressecção ou revisão endoscópica, coloração de mucosa a fim de identificar limites de lesão, magnificação de superfícies para melhor caracterização ${ }^{1,2,3}$, este se tornou o método principal na busca do diagnóstico das doenças colorretais, principalmente tumores e suas lesões precursoras, os pólipos ${ }^{1,3,4}$.

Considerando isso, o uso da colonoscopia tem aumentado no decorrer dos anos acompanhado pelo aumento da incidência de complicações ${ }^{5}$, apesar de ser exame invasivo seguro ${ }^{1}$. Mesmo com este aumento, a literatura considera que o risco é menor que o ganho que o exame traz na investigação de tumores colorretais e lesões precursoras ${ }^{5,6}$. Porém a literatura é, por vezes, benevolente com as complicações menos

Trabalho realizado na Disciplina de Coloproctologia do Departamento de Cirurgia da Irmandade da Santa Casa de Misericórdia de São Paulo - Faculdade de Ciências Médicas da Santa Casa de São Paulo. 
graves do exame colonoscópico, deixando-as a parte da avaliação da morbidade.

Existem vários fatores que modificam os índices de morbidade no exame colonoscópico tais como idade, comorbidades, se houve realização de procedimentos, o local da lesão submetida ao procedimento, as características dela, o tipo de procedimento, sedação, preparo de cólon, entre muitos outros 1,2,4,5,6,7,8,9.

Em vista disso, o objetivo do trabalho é avaliar os fatores de risco que determinam morbidade ao exame de colonoscopia.

\section{MÉTODO}

Cento e setenta pacientes consecutivos submetidos a exame colonoscópico na Área de Colonoscopia da Disciplina de Coloproctologia do Departamento de Cirurgia da Irmandade da Santa Casa de Misericórdia de São Paulo (Faculdade de Ciências Médicas da Santa Casa de São Paulo), no período de março a junho de 2009 foram analisados prospectivamente.

Todos os pacientes foram avaliados pela mesma equipe (residentes do serviço supervisionados pelos instrutores - todos autores do trabalho) com critérios previamente estabelecidos a fim de caracterizar fatores de risco para maior morbidade durante exame colonoscópico: idade, sexo, indicação, exame ambulatorial ou internado, efeitos adversos do preparo intestinal, qualidade do preparo, procedimento realizado durante colonoscopia e diagnóstico do exame. A morbidade foi caracterizada como intercorrências antes, durante ou após exame colonoscópico.

Os doentes se originam do Ambulatório de Colonoscopia da Santa Casa de São Paulo através de pedidos de colonoscopia solicitados por toda Irmandade (Hospital Central e todo complexo de hospitais satélites) além de Unidades Básicas de Saúde de nossa referência. Neste ambulatório, o médico avalia se há condições clínicas e suporte familiar de preparo domiciliar, quais as medicações habituais, orienta preparo intestinal, como o exame é realizado, suas possíveis complicações e alerta quanto a medicações que devem ser suspensas. Pacientes com comorbidades graves como insuficiência coronariana e insuficiência cardíaca congestiva classe funcional III e IV são internados na véspera do exame para preparo intestinal hospitalar. Pacientes com estomias desfuncionalizantes e em uso de cumarínicos também são internados para preparo colônico.
O preparo intestinal realizado em nosso serviço é com lactulose a 13\%: dieta sem resíduos os dois dias que precedem o exame, $20 \mathrm{mg}$ de bisacordil na véspera, $120 \mathrm{~mL}$ de lactulose com $500 \mathrm{~mL}$ de diluente (água, chá sem açúcar ou suco coado) ingeridos em trinta minutos de 6 a 8 horas antes da realização do exame. Pacientes com estomia desfuncionalizante são submetidos a clister glicerinado para limpeza colônica. Os pacientes internados cujo preparo anterógrado não foi satisfatório são submetidos a clister glicerinado como complementação.

A técnica do exame seguiu a padronização do serviço: drogas sedativas e analgésicas (midazolan e meperidina como padrão e propofol quando necessário), visando sedação consciente apropriada. Os exames foram realizados por vários endoscopistas (instrutores, residentes de coloproctologia, residentes de endoscopia, estagiários), mas a avaliação da morbidade foi avaliada apenas pelos autores.

Os fatores de risco (idade, sexo, exame ambulatorial ou internado, efeitos adversos do preparo intestinal, qualidade do preparo e procedimento realizado durante colonoscopia) foram comparados a morbidade através do programa Eviews com regressão não linear, considerando estatisticamente significante $\mathrm{p}<0,05$.

\section{RESULTADOS}

A amostra estudada apresenta pequena predominância do sexo feminino com 56,47\% (96 pacientes) e $43,53 \%$ do sexo masculino (74 pacientes). A média de idade foi 60,16 anos com desvio padrão $( \pm)$ de 14,69 anos. A tabela 1 caracteriza a faixa etária dos

Tabela 1 - Pacientes por faixa etária.

\begin{tabular}{lcc}
\hline $\begin{array}{l}\text { Década } \\
\text { de vida }\end{array}$ & $\begin{array}{c}\text { Número de } \\
\text { pacientes }\end{array}$ & Porcentagem \\
\hline Segunda & 01 & 0,59 \\
Terceira & 06 & 3,53 \\
Quarta & 09 & 5,29 \\
Quinta & 24 & 14,12 \\
Sexta & 31 & 18,24 \\
Sétima & 44 & 25,89 \\
Oitava & 46 & 27,05 \\
Nona & 08 & 4,70 \\
Décima & 01 & 0,59 \\
\hline
\end{tabular}


examinados, com predominância da sétima e oitava décadas.

As indicações dos exames estão relacionadas na tabela 2, evidenciando que as maiores indicações são exames de seguimento pós-operatório $(15,39 \%)$, presença já conhecida de pólipos ou seu seguimento (10,59\%) e presença já conhecida de tumores $(9,41 \%)$, respectivamente.

Três exames foram inconclusivos por mau preparo. Do restante, $36,53 \%$ foram normais. A maioria dos alterados apresentou pólipos $(26,95 \%)$, sendo destes $55,55 \%$ adenomatosos e $4,44 \%$ (dois casos) evidenciando adenocarcinoma. A tabela 3 mostra todos os achados endoscópicos.

Tabela 2 - Indicações do exame colonoscópico.

\begin{tabular}{lcc}
\hline Indicação & $\begin{array}{c}\text { Número } \\
\text { de }\end{array}$ pacientes & Porcentagem \\
\hline Alteração ritmo intestinal & 16 & 9,41 \\
Anemia & 07 & 4,12 \\
Diarréia & 08 & 4,70 \\
Doença Inflamatória (DII) & 05 & 2,94 \\
Dor abdominal & 11 & 6,47 \\
Exame rastreamento & 15 & 8,82 \\
Sangramento & 13 & 7,65 \\
Obstipação & 10 & 5,88 \\
Tumor & 16 & 9,41 \\
Pólipo & 18 & 10,59 \\
Pós-operatório & 26 & 15,30 \\
Rastreamento & 09 & 5,30 \\
Outros & 16 & 9,41 \\
\hline
\end{tabular}

Tabela 3 - Achados endoscópicos.

\begin{tabular}{lcc}
\hline Diagnóstico & $\begin{array}{c}\text { Número de } \\
\text { pacientes }\end{array}$ & Porcentagem \\
\hline Divertículo & 16 & 9,58 \\
Estenose* & 07 & 4,19 \\
Inflamação & 08 & 4,79 \\
Pólipo & 45 & 26,95 \\
Tumor** & 22 & 13,17 \\
Normal & 61 & 36,53 \\
Outros*** & 08 & 4,79 \\
\hline
\end{tabular}

* de anastomose ou secundária a DII.

** primário colorretal ou invasão extrínseca.

*** angiodisplasia, lipoma e úlcera solitária.
Cento e quarenta pacientes fizeram exames ambulatoriais $(82,35 \%)$ e o restante, internado com média de 3,73 $\pm 2,38$ dias de internação.

Quanto as comorbidades, 48,82\% dos pacientes possuíam alguma comorbidade, sendo Hipertensão Arterial Sistêmica a mais prevalente $(84,34 \%)$.

Apenas 22,94\% dos pacientes apresentaram algum efeito adverso ao preparo, sendo este considerado como cólica abdominal, náusea ou vômito.

Considerando a classificação de qualidade de limpeza intestinal utilizada por Fonoff ${ }^{10} \mathrm{em}$ Excelente, Boa, Regular e Má, relacionamos os pacientes dessa maneira (Tabela 4) e consideramos preparo limpo aqueles com excelente e bom preparo e sujo os demais (limpeza regular e má). Foram $65,88 \%$ de preparo limpo.

Foram realizados procedimentos em $44,7 \%$ dos exames, sendo a maioria polipectomias $(56,58 \%)$ entre biópsias, cauterizações, tatuagens e dilatações.

A morbidade chegou a 16,47\%, com 28 pacientes que sofreram alguma intercorrência peri-procedimento, perfazendo 34 intercorrências, conforme tabela 5. A morbidade se traduziu por complicações leves, sendo a desidratação a mais prevalente $(41,18 \%)$.

Tabela 4 - Qualidade do preparo de cólon.

\begin{tabular}{lcrl}
\hline Preparo & $\begin{array}{c}\text { Número de } \\
\text { pacientes }\end{array}$ & Porcentagem & \\
\hline Excelente & 60 & $35,29 \%$ & LIMPO \\
Bom & 52 & $30,59 \%$ & \\
Regular & 44 & $25,88 \%$ & SUJO \\
Ruim & 14 & $8,24 \%$ & \\
\hline
\end{tabular}

Tabela 5 - Morbidade do exame colonoscópico.

\begin{tabular}{lcc}
\hline Intercorrência & Número & Porcentagem \\
\hline Bradicardia* & 03 & $8,82 \%$ \\
Desidratação & 14 & $41,18 \%$ \\
Distensão & 07 & $20,60 \%$ \\
Peritonite & 02 & $5,88 \%$ \\
Sangramento & 05 & $14,70 \%$ \\
Suboclusão & 01 & $2,94 \%$ \\
Hipóxia & 01 & $2,94 \%$ \\
Descompensação & & \\
comorbidade** & 01 & $2,94 \%$ \\
Total & 34 & $100 \%$ \\
\hline
\end{tabular}

* com necessidade de atropina durante procedimento

** crise hipertensiva 
Não houve mortalidade. Porém ocorreram quatro complicações graves. Dois pacientes evoluíram com peritonite, um após polipectomia com pólipo grande $(3 \mathrm{~cm})$ cujo estudo anátomo-patológico mostrou se tratar de adenocarcinoma e o outro após preparo intestinal com dor, distensão, vômito e desidratação, além de exame inconclusivo por mau preparo devido tumor estenosante de sigmoide. Ambos foram submetidos a laparotomia exploradora, o primeiro com sutura do seguimento perfurado e transversostomia derivativa e o segundo com retossigmoidectomia do tumor abscedado e colostomia a Hartmann. Evoluíram bem, porém o primeiro descompensou comorbidades (arritmia) o que prolongou tempo de internação, desenvolvendo pneumonia nosocomial. A terceira complicação grave foi enterorragia pós polipectomia, necessitando nova colonoscopia para cauterização. E, por fim, a quarta complicação foi suboclusão por estenose secundária a Doença de Crohn, melhorada com tratamento clínico (jejum, analgesia, sonda nasogástrica e hidratação).

Todos os pacientes relatados fizeram exame ambulatorial, apenas o quarto foi encaminhado a internação imediatamente após o exame. Os pacientes de peritonite e enterorragia voltaram ao Pronto Socorro com tais queixas. Outros três pacientes foram internados após colonoscopia ambulatorial, devido dificuldade técnica durante exame e polipectomias difíceis, porém nenhum apresentou intercorrências no período de observação.

Assim, quando comparados idade, sexo, exame ambulatorial ou internado, efeitos adversos do preparo intestinal, qualidade do preparo e procedimento realizado durante colonoscopia com a morbidade apresentada na tabela 5, houve significância estatística apenas na qualidade do preparo e na realização de procedimento endoscópico. Valores estatísticos são apresentados na tabela 6.

\section{DISCUSSÃO}

As indicações para realização de exame colonoscópico neste trabalho diferiu da literatura devido as características do nosso serviço. Por ser um serviço quaternário e organizado pela Disciplina de Coloproctologia, muitos pacientes já vem para realizar procedimentos terapêuticos ou repetir exames para programar procedimentos cirúrgicos, o que explica o elevado índice de colonoscopias alteradas $(63,47 \%)$.
A literatura mostra sangramento e anemia, seguida de alteração do hábito intestinal como as principais indicações ${ }^{1}$, enquanto nosso estudo mostrou maior prevalência para exames de seguimento pós-operatório, presença já conhecida de pólipos (colonoscopias realizadas em outros serviços em que pólipos não foram removidos) ou seu seguimento e presença já conhecida de tumores (tumores tocáveis em busca de lesão sincrônica ou tomografia computadorizada de abdome sugerindo tumor colorretal).

Porém quanto ao diagnóstico, nosso estudo corrobora com a literatura com a maioria dos exames alterados evidenciando pólipos ${ }^{1}$,predominantemente adenomatosos o que ilustra a importância do exame endoscópico na prevenção do tumor colorretal ao encontrar lesões precursoras.

A morbidade geral deste estudo foi $16,47 \% \mathrm{com}$ $0 \%$ de mortalidade. Na literatura as taxas de morbidade variam de $0,2 \%$ nas colonoscopias diagnósticas a 1,2\% nas terapêuticas ${ }^{1}$, sendo perfuração responsável por apenas $0,1 \%$ e sangramento por $0,2 \%{ }^{1,4,5}$. Para Nahas et al. os índices de sangramento chegaram a $2 \%$ e de perfuração $0,5 \%$ após polipectomias.

O nosso estudo amplia a caracterização de morbidade da colonoscopia a fim de determinar todos os eventos mórbidos decorrente dela e de seu preparo. A literatura habitualmente caracteriza morbidade como complicação grave que não levou o paciente ao óbito e mortalidade aquela cujo êxito foi letal. Já as demais morbidades como desidratação, distensão abdominal, bradicardia, hipóxia entre outras, apesar de na prática clínica serem mais frequentes, são pouco citadas dificultando a análise real da morbidade do exame.

Tabela 6 - Fatores de risco versus morbidade: avaliação estatística.

\begin{tabular}{lc}
\hline Fator de risco & $\mathbf{p}^{*}$ \\
\hline Idade & 0,720 \\
Sexo & 0,695 \\
Ambulatorial/Internado & 0,937 \\
Comorbidades & 0,584 \\
Efeitos adversos & 0,208 \\
Qualidade do preparo & 0,007 \\
Procedimento & 0,006 \\
\hline
\end{tabular}

$* p<0,05:$ estatisticamente significante. 
Como o exame colonoscópico é amplamente indicado na atualidade, a análise de todos os eventos que o paciente pode sofrer é fundamental. A morbidade alcançada em nosso trabalho é o valor real, e não negligenciado, da realidade do nosso serviço, em que incluímos desde desidratação, passando por sangramentos auto limitados (ou clipados) durante o procedimento, distensão após o procedimento que levou o doente a observação/analgesia até as complicações graves como peritonite por perfuração e enterorragia. Considerando apenas os eventos graves do nosso estudo, temos $2,35 \%$ de morbidade, com $1,17 \%$ de perfuração e $0,6 \%$ de sangramento.

Com relação a sedação, a complicação mais frequente é a hipóxia podendo ocorrer em algum grau entre $17 \%$ e $70 \%$ dos doentes, tornando-se fator decisivo na interrupção do exame como o ocorrido em $0,42 \%$ dos casos no estudo de Nahas et al. e 2,94\% (um caso) no atual estudo.

Outro parâmetro que determina a progressão e eficácia do exame é o preparo de cólon. No nosso estudo, houve relação direta do preparo de cólon com a mordidade. Isso ilustra a fundamental importância de um bom preparo intestinal, particularizando-o para cada paciente a fim de diminuir as complicações que advém de um preparo inadequado. Quanto mais sujo o cólon, mais trabalhoso é o exame, a mucosa colônica é visualizada parcialmente podendo não diagnosticar lesões (pólipos e lesões planas, principalmente) e há maior chance de complicações.
A relação entre o aumento da idade dos pacientes submetidos ao método e a morbidade não apresentou valor estatisticamente significante, diferentemente do encontrado na literatura que apresenta relação direta com aumento de risco de eventos cardiovasculares e demais complicações após colonoscopia ${ }^{7}$, porém não diferente da população com mesma idade não submetida ao exame ${ }^{5}$.

O sangramento após colonoscopia tem relação direta com procedimento diagnóstico como biópsia e terapêutico como polipectomia ${ }^{1,2,5,6,8,9,11,12}$, assim como evidenciado no atual estudo. Este sangramento pode ocorrer durante o procedimento (quatro casos no presente estudo) ou mesmo depois de dias ${ }^{2,7,8,9,11}$ (um caso) e tem maior incidência quanto maior o pólipo ressecado ou sua característica séssil ${ }^{1,6}$.

A perfuração apesar de menos prevalente, apresenta elevado risco ao paciente, podendo ser letal. Ela ocorre predominantemente em pacientes com doença diverticular e obstrução ${ }^{7,9} \mathrm{e}$, principalmente, no cólon sigmóide e reto ${ }^{7,8,9,12}$. Os dois pacientes perfurados de nossa amostra foram no sigmoide por obstrução e pós polipectomia. Nosso estudo mostrou relação direta entre procedimento e morbidade, corroborando com a literatura.

\section{CONCLUSÃO}

A qualidade do preparo intestinal e a realização de procedimento endoscópico são fatores diretamente relacionados a morbidade do exame de colonoscopia.

\footnotetext{
ABSTRACT: Objective: The objective of this study is to analyze risks factors of morbidity on colonoscopy. Method: From March to June of 2009, 170 patients were examined and analyzed prospectively. Risks factors as age, sex, indication of exam, ambulatory or hospital exam, adverse events and quality of intestinal preparation, endoscopic procedure, diagnoses and incident before, during or after procedure were analyzed. Results: The average age was $60,16 \pm 14,69$ years old, with majority of female. The prevalent indication of exam was post-operative. Three exams were inconclusive due to bad colonic preparation. From the rest, 36,53\% were normal exams and the majority of the abnormal ones were polyps, adenomatous predominantly. In relation of morbidities, $\mathbf{4 8 , 8 2 \%}$ of patients present some morbidity, Arterial Hypertension as the most common. Only $\mathbf{2 2 , 9 4 \%}$ of patients have been some adverse event with the intestinal preparation. Colonic preparation was clean on $65,88 \%$ of exams and when compared with morbidity there were significant relation. Other significant factor was the presence of endoscopic procedures $(44,7 \%$ of exams), the majority polypectomies. Morbidity was $16,47 \%$, with dehydration more prevalent. There was no mortality. Conclusion: Quality of intestinal preparation and endoscopic procedures are factors directed related with colonoscopy morbidity.
}

Key words: Colonoscopy, Adverse events, Morbidity, Patient satisfaction. 


\section{REFERÊNCIAS}

1. Nahas SC, Marques CFS, Araújo SA, Aisaka AA, Nahas CSR, Pinto RA, Kiss DR. Colonoscopia como método diagnóstico e terapêutico das moléstias do intestino grosso: análise de 2.567 exames. Arq Gastroenterol. 2005;42(2):77-82.

2. Martínez MTG, Poblador AR, Raposo LG, Fernández AMG, Núñez JRC. Perforación tras colonoscopia: experiencia em 16 años. Rev Esp Enferm Dig. 2007;99(10):588-592.

3. Klug WA, Sampaio Neto P, Fonoff AM, Fang CB, Candelária PA, Capelhuchnik P. Preparo do Intestino para Colonoscopia com Lactulona a 8\%: Modo da Santa Casa de São Paulo. Rev bras Coloproct. 2008;28(1):084-088.

4. Swaroop VS, Larson MV. Colonoscopy as a screening test for colorectal cancer in average-risk individuals. Mayo Clin Proc. 2002;77:951-956.

5. Warren JL, Klabunde CN, Mariotto AB, Meekins A, Torpor M, Brown ML, Ransohoff DF. Adverse events after outpatient colonoscopy in the medicare population. Ann Intern Med. 2009;150(12):849-858

6. Consolo P, Luigiano C, Strangio G, Scaffidi MG, Glacobbe G, Giuseppe GD, Zirilli A, Pamiliart L. Efficacy, risk factors and complications of endoscopic polypectomy: Ten year Experience at a single center. World J Gastroenterol. 2008;14(15):2364-2369.

7. Levin TR, Zhao W, Conell C, Seeff LC, Manninen DL, Shapiro já, Schulman J. Complications of colonoscopy in an integrated health care delivery system. Ann Intern Med. 2006;145:880886.
8. Jentshura D, Raute M, Winter T, Kraus M, Manegold BC. Complications in endoscopy of the lower gastrointestinal tract. Surg Endosc. 1994;8:672-676.

9. Gatto NM, Fruncht H, Sundararajan V, Jacobson JS, Grann VR, Neugut AI. Risk of perforation after colonoscopy and sigmoidoscopy: A population-based study. J Natl Cancer Inst. 2003;93(3): 230-235.

10. Fonoff AM. Estudo comparativo entre solução oral de manitol e lactulose no preparo do cólon para colonoscopia. Tese de mestrado apresentada a Faculdade de Ciências Médicas da Santa Casa de São Paulo. 1996; São Paulo.

11. Wilkins T, LeClair B, Smolkin M, Davies K, Thomas A, Taylor ML, Strayer S. Screening colonoscopies by primary care physicians: A meta-analysis. Ann Family Med. 2009;7(1):56-62.

12. Lüning TH, Keemers-Gels ME, Barendregt WB, Tan ACLTL, Rosman C. Colonoscopic perforations: a review of 30,366 patients. Surg Endosc. 2007;21:994-997.

\section{Endereço para correspondência:}

FERNANDA BELLOTTI FORMIGA

Avenida Líbero Badaró, 1208 - Jardim São Caetano

São Caetano do Sul - SP

09581-610

Tel.: (11) 8187-7081 / (11) 4238-9739

E-mail: fformiga@colband.com.br 